\title{
Teamwork in chronic pain management and the way forward in low and middle-income countries
}

\author{
Ali Sarfraz Siddiqui ${ }^{1}$, Usman Bashir 2 \\ 1- Department of Anesthesiology. Aga Khan University, Karachi, Pakistan \\ 2- National Hospital and medical Centre, Defense Housing Authority phase 1, Lahore, Pakistan. \\ Correspondence: Dr. Ali Sarfraz Siddiqui, Flat\# L-7 Hassan Apartment Block, 13-A Gulshan-e Iqbal, Karachi, Pakistan; \\ E-mail: Sarfraz.siddiqui@aku.edu; Phone: +92 2134864397
}

\section{Abstract}

Assessment and management of patients with chronic pain is a challenging task due to its complex multidimensional nature and biopsychosocial impact. It requires a collaborative effort by an interdisciplinary team. Evidence showed that in low and middle-income countries (LMICs), 33\% patients among general adult population, and 56\% of general elderly population, suffer from chronic painful conditions. Women are more likely to report chronic pain than men. In this narrative review, we attempted to search published literature to know the status of assessment and management of patients with chronic pain in low resource countries and to know the most appropriate chronic pain management strategies used in developed countries. We used broad electronic database (January 2010- December 2020) through common search engines.

Lack of trained pain physicians and staffs, lack of interest, empathy and lack of integrated multidisciplinary approach are some of the barriers to achieve teamwork in chronic pain management. Authors strongly recommend the need of further research in this area, improvement in training programs for physicians and nursing staff for proper pain assessment and management of patients with chronic pain in low resource countries.

Key words: Chronic pain management; Teamwork; Low resource; Multidisciplinary team; Interdisciplinary approach; Low and middle-income countries

Citation: Siddiqui AS, Usman B. Teamwork in chronic pain management and the way forward in low and middleincome countries. Anaesth pain intensive care 2021;25(2):229-235. DOI: 10.35975/apic.v25i2.1477

Received: 18 January 2021, Reviewed: 20 January, 10 February 2021, Accepted: 9 March 2021

\section{Introduction}

Chronic pain is the pain that is present for more than three months. Osteoarthritis, low back pain, fibromyalgia, headaches, and neuropathy are common conditions that causes chronic pain. Persistent pain causes changes in perception and threshold for pain, social activities, and over all affects the quality of life. ${ }^{1}$ Prevalence of chronic pain increases with age and women are more likely to report chronic pain than men. ${ }^{2}$ Recent literature showed the prevalence of chronic pain in the range of $18 \%$ to $50 \%$ of adult patients with comparatively high prevalence in female patients. $^{3}$

Chronic pain has been recognized as a complex multidimensional condition with biopsychosocial consequences. A single treatment approach by one specialty therefore may not be sufficient for appropriate management of patient. This forms the basis for the need of well-integrated teamwork in managing patients with chronic pain. In developed countries, teamwork seen in routine clinical practice and is often strongly backed up by their National standards. Core standards for pain management 
services in the UK (CSPMS) include this statement as one of their core standards that "no sole practitioner acting in isolation, whatever their profession, can claim to run a pain management clinic or service". 4

Low and middle-income countries (LMICs), on the other hand face many challenges when it comes to delivery of chronic pain services as developing well integrated team. Access to proper pain management facilities is not freely available to a large proportion of population due to lack of infrastructure, public awareness, and appropriately trained healthcare providers. General population of developing countries present a huge burden of chronic illness where chronic pain shares a major proportion. Prevalence of chronic pain in LMICs is $33 \%$ in the adult patients, $56 \%$ in the older adults, and $35 \%$ in workers. ${ }^{5}$

Evidence suggests that the prevalence of chronic pain is under-estimated, and that treatment offered is inadequate. The inequities seen in low resource countries unfortunately include a disparity in the frequency and inability to treat pain in several different settings. Healthcare resources, regulation, educational needs and lack of integrated teams to treat distressing symptoms of chronic pain is disproportionately high in low resource environments. ${ }^{6}$ Rationale of this current review is to learn from the experience of the developed world regarding chronic pain management teamwork and to improve chronic pain management services in LMICs.

Our objectives were to review the published literature to know the chronic pain teamwork dynamics in the developed world, assess barriers and challenges in relation to teamwork in low and middle-income countries and suggest way forward keeping in view our existing infrastructure and lack of health care facilities.

\section{Search methodology}

We used broad electronic database (during January 2010 - December 2020) through search engines of PubMed, Google Chrome, Google Scholar, Cochrane library and Up-to-Date with literature in English language. Ten key words / terms were used to identify all relevant studies considering the overall objectives of the review.

With consensus of both authors, we included studies of any design mentioning chronic pain management, teamwork, low and middle-income countries, low resource countries, multidisciplinary, Interdisciplinary approach pain management program for adult patients and provided relevant information to address our review objectives. We excluded studies that mentioned pediatric patients and acute pain management practices.

\section{Importance of teamwork in chronic pain management}

Chronic pain alters pain perception and threshold for pain, social activities, and over all affect's quality of life. Chronic non-malignant pain may cause significant functional disability that have biopsychosocial consequences. It can alter sleep patterns, ability to exercise, undertake routine tasks, and social activities. Biopsychosocial model recognizes chronic pain conditions as a combination of physical dysfunction, beliefs and coping strategies, distress, disease behavior and social interactions. ${ }^{7}$ Due to these complex multi-dimensional aspects of chronic pain, a single treatment approach through one specialist may not be sufficient for the appropriate pain management of patient. ${ }^{8}$ Therefore biopsychosocial approach focuses on multidisciplinary approach, using various strategies targeting pain reduction, improving quality of life, mobility, psychological wellbeing and avoiding secondary dysfunction. To achieve these targets, long-term management plan is needed involving specialist treatments from a team which are tailored to the individual patient need in a wellintegrated manner. This forms the basis of a comprehensive pain management team. ${ }^{9}$

Multidisciplinary treatment is the multimodal treatment approach offered by health professional from different specialties. All these professionals provide health services on individual basis having own treatment goals for the patient and may not be coordinating with each other. Interdisciplinary treatment is the multimodal treatment offered by a multidisciplinary health care team coordinating in assessment and treatment plan using combined treatment goals. Team members coordinate with each other and discuss management plan in regular meetings. ${ }^{10}$ 


\section{Interdisciplinary approach to pain management}

Interdisciplinary approach encompasses medical management, behavioral, physical and psychological therapies, relaxation techniques, vocational therapy and pain education by a multidisciplinary team. Interdisciplinary team structure varies from country to country and region to region; however, in most of developed countries, routinely, the core team include physicians (primary care physician, pain physician and psychiatrist) and non-physicians (psychologist, physiotherapist and nurse). A combined and coordinated effort is vital to facilitate effective communication between all team members ${ }^{11,12}$

Chronic pain management is a significant and costly issue. Recent data supports the cost-effectiveness of interdisciplinary treatments for chronic painful conditions. Creating an interdisciplinary service can be quite difficult compared with the ease of simply colocating multiple services within one area. Once established, however, these interdisciplinary programs greatly enhance the effectiveness of treatment for the chronic pain sufferer and create a rewarding experience for the chronic pain team. ${ }^{13}$

\section{Roles and responsibilities of team members ${ }^{6}$}

\subsection{Primary Care Physician}

They play a central key role in long term management and to implement the pain management plan devised by the team and coordinate treatment strategy by effective feedback communication between various disciplines. Their role is also vital for review, regulation and control of opioid based medications prescribed for chronic pain.

\subsection{Anesthesiologists / Pain physicians}

Patients referred to specialist pain management center are assessed by pain physician who makes a comprehensive pain management plan incorporating a biopsychosocial approach. Pain physician has a central role in multidisciplinary team in that he/she then engages other members of the team according to individual patient needs and also brings the complex cases to multidisciplinary team meetings. They also recommend pain intervention procedures like peripheral or central nerve blocks, chemical or radiofrequency nerve ablation procedures, device implantation and other pain management modalities.

\subsection{Physiotherapist}

They are invaluable members of the team and have important contributions through their health promotion, prevention, and assessment and treatment activities. They play vital roles in restoring and improving functional mobility of patients.

\subsection{Specialized Nurses}

They are actively involved in assessments of the patient's pain and helps to establish treatment plans and provide assistance in interventional pain procedures. They also communicate and coordinate with patients and provides ongoing patient education.

\subsection{Psychologist}

They are responsible for day-to-day psychosocial care of the patient. They provide input through various therapies for example cognitive behavioral therapy, acceptance and commitment therapy biofeedback techniques and hypnosis are few of important ones regarding pain management.

\subsection{Psychiatrists}

Patients with chronic pain may have psychiatric issues. If any such issue is picked by primary physician or pain team, then they should be referred to psychiatrists first for proper management of variety of psychiatric disorders, like anxiety, depression, addiction, and post-traumatic stress disorder so that they can benefit from pain team's input.

\subsection{Pharmacist}

They may be contacted by primary care physician in making management plan for patients with complex issues like drug interactions, misuse or risk of overdose.

\subsection{Dieticians}

Chronic pain patients are usually older adults and have dietary issues, and lack of appetite. Such patients need special advice on nutrition by dietician.

Various other medical specialists and healthcare providers are involved as part of interdisciplinary team from time to time through a coordinated approach 
depending upon individual patient's needs. More common of these team members include Neurosurgeon, Orthopedic surgeon, neurologist, rheumatologist, Occupational therapist, and Social worker.

\section{Advantages of the multidisciplinary care ${ }^{14}$}

Multidisciplinary pain management is a clinically efficient and cost- effective method than usual care. Following are few advantages of this approach;

i. This allows appropriate diagnosis of chronic pain condition

ii. Prevents duplication of investigations

iii. Rapid initiation of treatment plan after diagnosis

iv. Various treatment options are made available

v. Programmed health care is provided in a coordinated manner vi. Evidence based and safe treatment options are provided

\section{Teamwork in clinical practice}

Team member work together closely by collaborating their assessments and treatment plans in regular multidisciplinary team meetings (MDTs). These meetings are focused on diagnosis, therapeutic aims and plans for treatment along with review of progress of more complex patients. These meetings can be face to face, online or combination of both. Frequency of meetings varies from center to center; however, in most centers regular team members meet at least once a week on a fixed day and time.

Pain management program is another example of wellcoordinated teamwork in chronic pain management. The team running the program includes clinical psychologist, physiotherapist, pain physician, specialist nurse and patient. They provide combination of cognitive-behavioral therapy and physical therapies

Table 1: Barriers towards teamwork in chronic pain management in low and middle-income countries ${ }^{20,21}$

\begin{tabular}{|c|l|}
\hline S. No. & Possible barriers \\
\hline 1 & Lack of clearly defined standards and Pain management protocols \\
\hline 2 & Poor access to pain specialists \\
\hline 3 & Shortage of trained specialists and healthcare professionals \\
\hline 4 & $\begin{array}{l}\text { Limited healthcare resource allocation from the Government (Pain management has low priority in } \\
\text { Government healthcare policies) }\end{array}$ \\
\hline 5 & Treatment by non-qualified unregistered practitioners \\
\hline 6 & Inadequate knowledge, Skills, and attitudes of healthcare professionals \\
\hline 7 & Lack of training and awareness to work as a team. \\
\hline 8 & $\begin{array}{l}\text { Lack of shared attitudes in clinicians and healthcare providers (lack of common agreed goals, } \\
\text { shared objectives) }\end{array}$ \\
\hline 9 & Lack of culture to facilitate a teamwork. \\
\hline 10 & False health beliefs \\
\hline 11 & Heavy workload and lack of time to work in collaboration as a team. \\
\hline 12 & Hierarchical work environment (exacerbated distinction between nursing staff and clinicians) \\
\hline 13 & Inappropriate organizational setup to promote teamwork. \\
\hline 14 & Unstable and inconsistent healthcare workforce \\
\hline 15 & $\begin{array}{l}\text { Competing environment for patient /client in private sector (individual clinicians and healthcare } \\
\text { workers working for fee for service) }\end{array}$ \\
\hline
\end{tabular}


Table 2: Recommendations and way forward for chronic pain management in $\mathrm{LMICs}^{22,23}$

\begin{tabular}{|c|l|}
\hline S. No. & Recommendations and way forward \\
\hline 1 & Promoting formal education and training opportunities of pain assessment and management \\
\hline 2 & $\begin{array}{l}\text { Establish Pain management as a recognized medical and nursing specialty through an official } \\
\text { national authority. }\end{array}$ \\
\hline 3 & Formal training in communication and teamwork (both undergraduate and post- graduate levels) \\
\hline 4 & $\begin{array}{l}\text { Including pain assessment and management a mandatory component of all medicine, nursing, } \\
\text { and undergraduate curricula }\end{array}$ \\
\hline 5 & Promoting culture of frequent, clear direct communication between healthcare team members \\
\hline 6 & Promoting collaborative approaches in clinical education, quality improvement and research \\
\hline 7 & $\begin{array}{l}\text { Incorporating pain management into the national health care agenda (Prioritizing Pain } \\
\text { management in health care planning, resources, financing and delivery) }\end{array}$ \\
\hline 8 & Develop national level pain management guidelines and standards. \\
\hline 9 & $\begin{array}{l}\text { Promoting a culture to reduce competition for patient by engaging clinicians as employees by } \\
\text { organizations (especially in private sector Institutes) }\end{array}$ \\
\hline 10 & Promoting a culture to reduce hierarchal work environment \\
\hline
\end{tabular}

in a group-based setting over a defined time period. Their input empowers patients with self-management strategies and improve function and quality of life. ${ }^{15}$

Many primary care physicians are reluctant to refer patients to specialized pain centers at the initial presentation that often delay proper care to patients with chronic pain. If referred timely, the pain management specialists may provide a more accurate diagnosis of painful condition and therefore, more effective therapeutic outcomes of these patients can be achieved. ${ }^{16}$ In a robust healthcare system, pain specialist clinics are an integral component of multidisciplinary pain management program and are more effective at assessing individual patient needs. Evidence-based research from the Pain Association of Singapore Task Force highlights the importance of these multidisciplinary teams for the effective administration of opioid therapy for chronic pain patients. $^{17}$

\section{Challenges in low resource countries}

Evidence based and effective chronic pain management is a major challenge for public health organizations and healthcare systems around the globe. Barriers related to physicians and health care staff included lack of awareness, interest and sympathy, expertise in pain assessment and management and lack of communication between healthcare professionals. Barriers related to healthcare system are lack of specialized pain care centers and pain specialists, short consultation time with the physicians, long waiting time for appointments in specialized centers and the lack of integrated multidisciplinary approach to pain management. ${ }^{18}$

Recent data is not available from the developing world but in low resource countries this problem is even higher with multiple challenges and barriers to the provision of teamwork in chronic pain. There is significant amount of variation in the quality and standards of healthcare provision in public and private sector hospitals. There is lack of awareness to public and trained healthcare providers. Because of other more common health issues, pain management is not amongst the priority in public sector hospitals. Barriers towards teamwork in chronic pain management in low and middle-income countries are presented in table 1 .

\section{Solutions to the challenges ${ }^{19}$}

Among LMICs, chronic pain management services can be improved by focusing on following three inter- 
dependent areas: advocacy, improving treatment availability, and education.

\subsection{Advocacy}

At the national and local level, we need to work collaboratively to develop the specialties of pain medicine. Organizations such as the International Association for the Study of Pain (IASP) and the World Federation of Societies of Anesthesiologists (WFSA) are working with national professional societies to develop local expertise and leadership. As part of creating awareness and advocacy, high priority should be given to monitoring the impact of interventions to improve pain management services in the country.

\subsection{Improving treatment availability}

Simple treatments can make a big difference but even these may not be available in many LMICs. Appropriate access to opioid medications and other analgesic treatments not only depends on legal and policy considerations, but also on the knowledge and attitudes of healthcare workers, hence the vital role of education in improving chronic pain management service.

\subsection{Education}

Effective pain management education underpins advocacy efforts, and improved availability and use of treatment options. Educational programs are required to improve knowledge and change attitudes to manage chronic pain.

As mentioned earlier, barriers to pain management are considerable, and include poor knowledge and attitudes about pain management, low prioritization of pain management by governments and hospitals, inappropriate legislation, and limited or non-existent availability of pain treatments. Proper pain education may play a central role in overcoming these barriers. We all need to work together to increase awareness, improve knowledge and attitudes, and make appropriate treatments available to achieve the best possible health for our patients with chronic pain.

\section{Practice}

\section{recommendations \& way forward}

Multidimensional public health measures (like social, educational, psychological, and behavioral) have been recommended to prevent the increasing prevalence of chronic pain. Lifestyle modifications like smoking cessation, weight reduction, stress-free behavior, and regular physical exercise can contribute to limit the development of chronic pain. With medical management, preventive measures, awareness and counseling session are also important in the management of chronic painful conditions.

The biopsychosocial model of pain implies that optimal management requires a multidisciplinary team approach with good communication, as well as input from a range of properly educated, specialist healthcare professionals. There is a need to recognize chronic pain as a disease, develop local or national guidelines for managing chronic non-cancer pain, and adopt patient-centered approach.

We believe that improvement of health care facilities in low and middle-income countries require a commitment to learn from the research of the same region. Authors strongly feel the need of research in this area, improvement in training programs for physicians and nursing staff and services for proper pain assessment, management and follow up of patients with chronic pain in low resource countries. Recommendations and way forward for better teamwork in chronic pain management in low and middle-income countries are presented in table 2.

\section{Conflict of Interest}

None declared by the authors

\section{Authors' Contribution}

SS: Literature review, data extraction, manuscript writing, practice recommendations

UB: Literature review, manuscript writing, manuscript review and editing 


\section{References}

1. Walters JL, Baxter $\mathrm{K}$, Chapman $\mathrm{H}$, Jackson $\mathrm{T}$, Sethuramachandran $A$, Couldridge $M$, et al. Chronic pain and associated factors in India and Nepal: a pilot study of the Vanderbilt global pain survey. Anesth \& Analg. 2017; 125(5):1616-26. DOI: $10.1213 / A N E .0000000000002360$

2. Gaskin DJ, Richard P. The economic costs of pain in the United States. J. Pain. 2012; 13:715-724. DOI: 10.1016/j.jpain.2012.03.009

3. Sá KN, Moreira L, Baptista AF, Yeng LT, Teixeira MJ, Galhardoni R, et al. Prevalence of chronic pain in developing countries: systematic review and metaanalysis. Pain Reports. 2019;4(6):779. DOI: $10.1097 / P R 9.0000000000000779$

4. Collet B and Rockett M. Definition, membership, and interaction of the multidisciplinary and multispecialty team. Core standards for pain management services in the UK. 2015. https://fpm.ac.uk/sites/fpm/files/documents/201907/Core $\% 20$ Standards $\% 20$ for $\% 20$ Pain $\% 20$ Manageme nt $\% 20$ Services.pdf

5. Jackson T, Thomas S, Stabile V, Han X, Shotwell M, McQueen K. Prevalence of chronic pain in low-income and middle-income countries: a systematic review and meta-analysis. The Lancet. 2015;385:S10. DOI: $10.1016 /$ S0140-6736(15)60805-4

6. Kress HG, Aldington D, Alon E, Coaccioli S, Collett B Coluzzi $F$, et al. A holistic approach to chronic pain management that involves all stakeholders: change is needed. Curr Med Res Opin. 2015;31(9):1743-54. DOI: $10.1185 / 03007995.2015 .1072088$

7. Kawi J, Reyes AT, Arenas RA. Exploring pain management among Asian immigrants with chronic pain: Self-management and resilience. J Immigr Minor Health. 2019;21(5):1123-36. DOI: 10.1007/s10903018-0820-8

8. Banerjee S, Argáez C. Multidisciplinary treatment programs for patients with chronic non-malignant pain: a review of clinical effectiveness, cost-effectiveness, and guidelines. Ottawa: CADTH 2017 (CADTH rapid response report: summary with critical appraisal). Ottawa (ON): Canadian Agency for Drugs and Technologies in Health; 2017.

9. Tompkins DA, Hobelmann JG, Compton P. Providing chronic pain management in the "Fifth Vital Sign" Era: Historical and treatment perspectives on a modern-day medical dilemma. Drug Alcohol Depend. 2017;173:S11S21. DOI: 10.1016/i.drugalcdep.2016.12.002

10. Nicholas M, Ushida T, Wallace M, Williams A, Wittink H, Edwards $\mathrm{R}$, et al. Task force on multimodal pain treatment defines terms for chronic pain care. https://www.iasppain.org/PublicationsNews/NewsDetail .aspx? ItemNumber=6981.

11. Kamper SJ, Apeldoorn AT, Chiarotto A, Smeets RJ, Ostelo RWJG, Guzman J, et al. Multidisciplinary biopsychosocial rehabilitation for chronic low back pain.
Cochrane Database Syst Rev. 2014;(9):CD000963. DOI:10.1002/14651858.CD000963.pub3.

12. Lewis GN, Bean D, Mowat R. How have chronic pain management programs progressed? A Mapping Review. Pain Pract. 2019;19(7):767-84. DOI: $10.1111 /$ papr.12805

13. Gatchel RJ, McGeary DD and McGeary CA and Lippe B. Interdisciplinary chronic pain management past, present and future. Am Psychol. 2014;69(2):119-30. DOI: $10.1037 / a 0035514$.

14. Joypaul S, Kelly F, McMillan SS, King MA. Multidisciplinary interventions for chronic pain involving education: A systematic review. Plos one. 2019;14(10):e0223306.

DOI: $\underline{10.1371 / j o u r n a l . p o n e .0223306}$.

15. Wilkinson $P$ and Whiteman R. Pain management programs. BJA CEPD Reviews. 2016;17(1):10-5. https://doi.org/10.1093/bjaed/mkw027

16. Cheung CW, Choo CY, Kim YC, Lin FS, Moon SH, Salido EO, et al. Collaborative efforts may improve chronic non-cancer pain management in Asia: findings from a ten-country regional survey. J Pain Relief. 2016;225. DOI:10.4172/2187-0846.1000225.

17. Yanni LM, McKinney-Ketchum JL, Harrington SB, Huynh C, Amin S, Matsuyama R et al. Preparation, confidence, and attitudes about chronic noncancer pain in graduate medical education. J Grad Med Educ. 2010;2(2):260-68. DOI: 10.4300/JGME-D-10-00006.1

18. Hadi MA, Alldred DP, Briggs M, Marczewski K, Closs SJ. Treated as a number, not treated as a person: a qualitative exploration of the perceived barriers to effective pain management of patients with chronic pain. BMJ open. 2017;7(6):1-7. DOI: 10.1136/bmjopen-2017$\underline{016454}$

19. Morriss WW and Roques CJ. Pain management in lowand middle-income Countries. BJA Educ. 2018;18(9):265-270. DOI: 10.1016/j.bjae.2018.05.006

20. Cartmill C, Soklaridis S and Cassiday JD. Transdisciplinary Teamwork: The experience of clinicians at a functional restoration program. J Occup Rehabil. 2011;21:1-8 . DOI: 10.1007/s10926-0109247-3

21. Stanos S. Focused review of interdisciplinary pain rehabilitation programs for chronic pain management. Curr Pain Headache Rep (2012) 16:147-152. DOI: 10.1007/s11916-012-0252-4

22. Al-Mahrezi A. Towards effective pain management: Breaking the Barriers. Oman Medical Journal. 2017;32:357-358. DOI: 10.5001/omi.2017.69

23. Felicia MK, Paul EF, Eric LK, Liliana DL, Afsan B, Xiaoxiao JK et al. Alleviating the access abyss in palliative care and pain relief- an imperative of universal health coverage: The Lancet Commission report, Lancet. Lancet. 2018;391:1391-454. DOI: $10.1016 /$ S0140-6736(17)32513-8 